\title{
Workshop on active learning: two examples
}

\section{Zohra Ben Lakhdar, Souad Lahmar, Vasudevan Lakshminarayanan}

Zohra Ben Lakhdar, Souad Lahmar, Vasudevan Lakshminarayanan, "Workshop on active learning: two examples," Proc. SPIE 9289, 12th Education and Training in Optics and Photonics Conference, 928929 (17 July 2014); doi: 10.1117/12.2070726

SPIE Event: 12th Education and Training in Optics and Photonics Conference, 2013, Porto, Portugal 


\author{
Workshop on Active Learning: Two Examples \\ Zohra Ben Lakhdar ${ }^{\mathrm{a}}$, Souad Lahmar*b ${ }^{*}$ asudevan Lakshminarayanan ${ }^{\mathrm{c}}$ \\ ${ }^{a}$ Department of physics-Faculty of Sciences - University Tunis el Manar-Tunisia \\ ${ }^{\mathrm{b}}$ Institut Préparatoire aux études Scientifiques et Techniques (IPEST), BP 51, 2070 La Marsa - TUNISIA, \\ ${ }^{\mathrm{c}}$ School of Optometry and Vision Science and Departments of Physics and Electrical and Computer Engineering, \\ Unviersity of Waterloo, Waterloo, ON N2L 3G1, Canada
}

\begin{abstract}
Optics is an enabling science that has far ranging importance in many diverse fields. However, many students do not find it to be of great interest. A solution to this problem is to train teachers in active learning methodologies so that the subject matter can be presented to generate student interest. We describe a workshop to present an example of an active learning process in Optics developed for training of teachers in developing countries (a UNESCO project) and will focus on $z$ two different activities: 1 . Interference $\&$ diffraction is considered by students as being very hard to understand and is taught in most developing countries as purely theoretical with almost no experiments. Simple experiments to enhance the conceptual understanding of these wave phenomena will be presented and 2. Image formation by the eye. Here we will discuss myopia, hyperopia and astigmatism as well as accommodation. In this module we will discuss image. The objective of the workshop will be to provide an experience of the use of the active learning method in optics including the use of experiments, mind's on \& hands-on exercises, group \& class discussions
\end{abstract}

Keywords: Active Learning, UNESCO, Geometrical Optics, lenses, visual optics, wave phenomena, interference, diffraction, interactive lecture demonstrations.

\title{
1. INTRODUCTION
}

The United States National Research Council in a famous report ${ }^{1}$ has called optics an "Enabling Science" and has far reaching technological implications, such as in biotechnology, nanoscience, energy, etc. Therefore, it is imperative that optics be taught (and taught well) to students who are the future technologists and scientists. In its efforts to promote creativity and innovations in the way introductory physics is taught in the university, UNESCO (United Nations Educational and Scientific Organization) has supported activities in different developing countries to address the need for teacher upgrading and introducing innovative learning approaches. In recent years, the focus has been active learning approach e.g., see references 2-7, including developing teaching and learning materials. The focus of the UNESCO Active Learning in Optics and Photonics project begun in 2003 is based on one of the experimental physics areas that is relevant and adaptable to research and educational conditions in many developing countries. This project was developed for the benefit of university and senior high school physics teachers from developing countries and aims to train and better equip them to teach the optics part of the introductory physics course by using active learning with hands-on activities with very simple and inexpensive homemade equipment and by drawing examples from local research activities.

What is active learning? The major difference between active learning and traditional methods is that active learning is a hands-on, minds-on activity. Active learning in physics, developed over the last two decades, has been demonstrated to enhance student understanding of basic physics concepts. In this learning model, students are guided to construct their knowledge of physics concepts by direct observations of the physical world. Use is made of a learning cycle including predictions, small group discussions, observations and comparison of observed results with predictions. In this way, students become aware of the differences between the beliefs that they bring into the classroom, and the actual physical laws that govern the physical world. An evolving product of many years of physics education research, the active learning method is known to measurably increase conceptual understanding. It reproduces the scientific process in the classroom and aids in the development of good physical reasoning skills. The reader is referred to the extensive literature on active learning. In this workshop we present two examples of the active learning methodology, adapted from the UNESCO ALOP project.

* email: soulahmar@yahoo.fr ; Phone:+216 29251 660; fax: +216 71746551 


\section{TWO OPTICS MODULES}

As noted above, all these activities require very simple and inexpensive equipment to teach optics concepts. These can include, ruler, meter stick, inexpensive laser pointers (can be purchased in Asian markets for less than \$1.00), cardboard screens, clay or putty (for mounting laser, cardboard screens, lenses, etc.). In fact, the most expensive pieces are lenses. However, a search of the web will provide contact information for lens suppliers in China who can supply pairs of simple spherical lenses for less than 50 cents. The main aim, is to keep costs down and the material simple. It should be noted that these activities work best when students work in small groups of 3 or 4 and are designed for use in the laboratory. The instructor should give plenty of time for students to discuss and talk amongst themselves.

\subsection{Optics of the Eye}

This module presents a very simple, introduction to image formation by the eye. The purpose is for the students to gain a conceptual understanding of the effects of different lenses using geometrical optics model and to see how these ideas apply to the normal functioning of the eye. Possible abnormalities of the eye are also considered. The eye contains two components that function as lenses: the cornea (the colored outer portion) and the crystalline lens ${ }^{8}$. In order to understand how the eye forms an image on the retina (the layer of light sensitive cells and accompanying neurons at the retina), the student will learn about imaging by simple spherical lenses (both diverging, negative and positive, converging lenses). In addition, he/she will form a simple model of the eye, consisting of a positive spherical lens and a "retina" at the focal point of the lens. This model will serve as the "normal" or emmetropic eye.

With this basic set up, the student will then set up a myopic eye (near sighted) and will learn what type of lenses will correct such a near sighted eye. In this case, the image will fall before the retina, and a negative lens will be used to bring the image to a focus. For a far sighted eye (hyperopia), the retina is close to the lens and the image focal point is behind the retina. In this case the student will discover that a positive lens will be used to correct for hyperopia.

The eye can also suffer from what is known as astigmatism - this is because the curvature of the eye (cornea) is not equal in all meridians, and therefore, the eye will be astigmatic. The student will investigate image formation by cylindrical lenses.

Lastly, the student will be able to investigate the mechanism of accommodation. This is the process in which the crystalline lens will change its curvature to make the whole eye power greater to bring to focus a near-by object. This will be investigated using the eye model. At the conclusion of this module, the student should be able to understand that the eye is not a fixed focus system, but is capable of varying its focus for far to near objects, and to understand the basic optics of myopia, hyperopia and how these refractive anomalies of the human eye are corrected by spectacles or contact lenses. Finally, the student will develop a good conceptual idea of image formation by cylindrical lenses.

\subsection{Interference and Diffraction}

This module deals with the behavior of light when it is incident on very small slits or obstacles ( of very small dimensions ). In order to understand the behavior of light under such conditions, we need to give up the geometrical optics analysis of light propagation and think of light in terms of wave phenomena?

With an inexpensive laser pointer it is possible to observe many different interference and diffraction patterns. The laser pointer is a good source of monochromatic coherent light which is needed for optimal demonstration of interference and diffraction effects. The students should be reminded of laser safety. By shining laser light coming from an inexpensive laser pointer through two narrow homemade slits, it is possible to observe an interference pattern (bright and dark fringes) on a distant screen. The slits could be made by scratching two very close and parallel thin lines on aluminum coating of a piece of mirror, using a razor blade (or Exacto knife). In fact, simple household items like buttons, or a think wire can be used to show diffraction. Assuming a wave model (and using eventually Interactive Lecture Demonstrations ${ }^{5,10}$ - ILDs; see figure 1) it is possible to explain, in a very simple way, the central part of the pattern in terms of superposition and interaction of two coherent light waves coming from the division of the incident laser wave front on the two slits ${ }^{11}$. 
On the other hand, the diffraction phenomena that happen when laser light is incident on a single narrow slit can be examined and explained, according to the Huygens principle, in terms of interference between waves originating from different points of the part of the same wave front that passes through the slit. The students are then able to understand the whole light pattern (not only the central part) observed in double-slit interference.
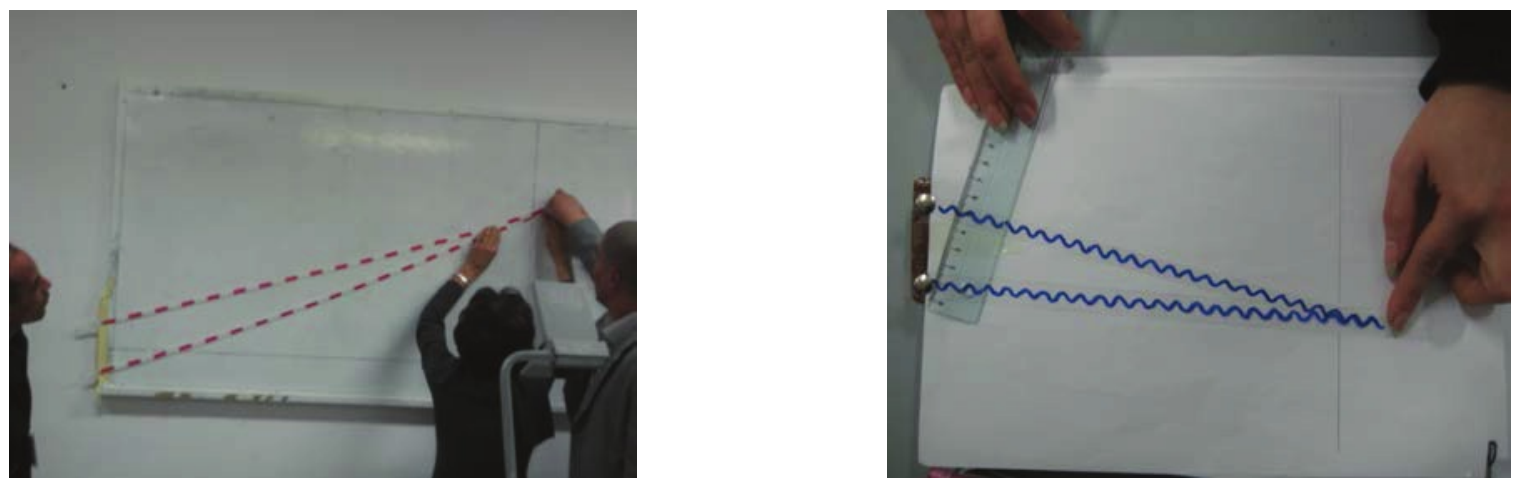

Figure 1: Example of Interactive Lecture Demonstration (left) and group activity (right) illustrating phase changes for light coming from two sources.

Finally, the pattern produced when coherent quasi-monochromatic light and white light are shone through a multiple parallel slits system called diffraction grating (a very important device in spectroscopy) can be observed and explained. Inexpensive diffraction grating can be fabricated by peeling off the $\mathrm{CD}$ coating (for a transmission grating) or reflection of a $\mathrm{CD}$ (reflection grating).

At the end of this module, the student should be able to observe and explain the behavior of light when light waves from coherent sources are superposed on each other and understand the concept of linear superposition. He/she should also be explain what happens when light interacts with an obstacle or slit and understand that the so called diffraction phenomena is due to the superposition of waves originating from points on the same wave-front division interference whereas the interference phenomena describes the result when a wave-front is divided into parts by passing through two or more distinct holes or slits. Most importantly, the student should have a clear idea of the wave nature of light.

\section{DISCUSSION AND CONCLUSIONS}

Active learning developed in the United States about 20 years ago, has been shown to enhance student understanding of basic physics concepts. The basic idea of the active learning strategy is the inclusion of predictions, small group discussions, observations and comparison of observed results with predictions. This learning cycle is often represented as PODS: Prediction, Observation, Discussion and Synthesis. There is considerable evidence that active learning method measurably improves conceptual understanding. This PODS process reproduces in the classroom the scientific process and aids in the development of good physical reasoning skills ${ }^{10}$.

The crucial fact is that in the active learning process, the instructor has a different role than in traditional processes. The instructor no longer lectures, but rather acts as a facilitator and guides in the learning process and is not the "authority". This is often a difficult transition and the temptation to lecture and be the authority figure is great. The instructor has to have faith in the ability of the active learning process to teach concepts. This transition also depends upon cultural factors and can vary from country to country. 
The two activities presented here are part of the UNESCO ALOP project which consists of 6 modules ${ }^{12}$ :

1. Introduction to Geometrical Optics

2. Lenses and Optics of the Eye

3. Interference and Diffraction

4. Atmospheric Optics

5. Optical Data Transmission

6. Wavelength Division Multiplexing.

These modules are intended for university and senior high school physics teachers from developing nations and aims to train and better equip them to teach the optics part of the introductory physics course using active learning with hands on activities using very simple and cheap materials.

What is next? These are introductory concepts - the active learning technique has to be extended to more complex concepts and quantitative learning methods. These could include the use of numerical simulations and modeling using easy to use software such as Mathematica (Wolfram Research, Urbana-Champaign, IL.) which is an ideal platform for doing simulations of physical phenomena. One of us (VL) has used Mathematica simulations for interactive lecture demonstrations and has found them to be very effective. The Wolfram Alpha is another ideal tool - this is an App that can be downloaded on smart phones and are very sophisticated. It is also possible to make attachments for smartphones to take and analyze data in real time and for modeling. These need to be investigated for future work.

\section{REFERENCES}

[1] National Research Council, Harnessing Light, National Academy Press, Washington DC, 1998.

[2] Alarcon, M., Ben Lakhdar, Z., Culaba, I., Lahmar, S., Lakshminarayanan, V., Mazzolini, A., Maquiling, J., Niemela, J. " Active learning in optics and photonics: a model for teacher training and professional development:, Proc SPIE, 77833, DOI: 10.1117/12.860708; http://spie.org/x648.html?product_id=860708 (2010)

[3]. Alarcon, M., Arthurs, E., Ben lakhdar, Z., Culaba, I., Lakshminarayanan, V., Maquiling, J., Mazzolini, A., Niemela, J., and Sokoloff, D., "Active learning in optics and photonics: achievements and outcomes to date", ETOP meeting, Ottawa, 2007.

[4]. ibid, "Active learning in optics and photonics: experiences in Africa", ETOP 2005 http://spie.org/etop/etop2005 040.pdf (2005)

[5] D. Sokoloff, P. Laws, and R. Thornton, "RealTime Physics: active learning labs transforming the introductory laboratory", European Journal of Physics 28: S83-S94 (2007).

[6] Masters, M.F., and Grove, T.T., "Active Learning in intermediate Optics through class tutorials and concept building laboratories", ETOP 2009, http://spie.org/etop/2009/etop2009 3b.8.2.pdf (2009)

[7] Lakshminarayanan V, "Interactive lecture demonstrations, active learning and the ALOP project", Eco-Photonics, Proc. SPIE 8065, doi: 10.1117/12.889508 (2011),

[8] Garhart C, and Lakshminarayanan, V., "Anatomy of the Eye", in J.Chen, W.Cranton and M. Fihn, Handbook of Visual Display Technology, Volume 1, Pages 73-83, Springer, Heidelberg,2012.

[9] Ghatak, K., Optics, McGraw-Hill, New York, 2012.

[10] Sokoloff, D, Thornton, RK, Laws, PW., RealTime Physics, John Wiley, Hoboken, NJ, 1997.

[11] R. Baierlein and V. Miglus, "Illustrating double slit interference: yet another way", Am. J. Physics, 59:857, (1991)

[12] Sokoloff, D. (Ed.), Active Learning in Optics and Photonics, Manual, UNESCO, Paris, 2006 